\title{
Density functional theory analysis of structural and electronic properties of orthorhombic perovskite $\mathrm{CH}_{3} \mathrm{NH}_{3} \mathrm{PbI}_{3}$
}

\author{
Yun Wang, ${ }^{a}$ Tim Gould, ${ }^{\mathrm{b}}$ John F. Dobson, ${ }^{\mathrm{b}}$ Haimin Zhang, ${ }^{\mathrm{a}}$ Huagui Yang, ${ }^{\text {a,c }}$ Xiangdong Yao, and Huijun \\ Zhao ${ }^{* a, d}$
}

${ }_{5}$ Received (in $\left.X X X, X X X\right)$ Xth $X X X X X X X X X 20 X X$, Accepted Xth $X X X X X X X X X 20 X X$

DOI: 10.1039/b000000x

The organic/inorganic hybrid perovskite $\mathrm{CH}_{3} \mathrm{NH}_{3} \mathrm{PbI}_{3}$ is a novel light harvester, which can greatly improve the solar-conversion efficiency of dye-sensitized solar cells. In this study, a first principle theoretical study is performed using local, semi-local and non-local exchange-correlation approximations

10 to find a suitable method for this material. Our results, using the non-local optB86b+vdWDF functional, excellently agree with the experimental data. Thus, consideration of weak van der Waals interactions is demonstrated to be important for the accurate description of the properties of this type of organic/inorganic hybrid materials. Further analysis of the electronic properties reveals that I $5 p$ electrons can be photo-excited to $\mathrm{Pb} 6 \mathrm{p}$ empty states. The main interaction between the organic cations and

15 inorganic framework is through the ionic bonding between $\mathrm{CH}_{3}$ and I ions. Furthermore, I atoms in the

$\mathrm{Pb}-\mathrm{I}$ framework are found to be chemically inequivalent because of their different chemical environments.

\section{Introduction}

20 Dye-sensitized solar cells (DSCs) have stood out among various photovoltaic devices owing to their low cost, simple fabricating procedure, environment friendliness, and relatively high efficiency. Consequently, DSCs are promising candidates for the next generation of solar cells. ${ }^{1-3}$ One recent breakthrough on ${ }_{25}$ DSCs is the introduction of organic/inorganic hybrid perovskite materials as novel light harvesters, such as $\mathrm{CH}_{3} \mathrm{NH}_{3} \mathrm{PbI}_{3}$, which can improve the solar conversion efficiency of DSCs to the current best record, ca. $15 \%{ }^{4-10}$ The high efficiency has been demonstrated to the long exciton diffusion lengths in the 30 perovskite materials. ${ }^{11-12}$ Therefore, understanding the structures and chemistries of these prototype light harvesters is important for the molecular design of organic/inorganic perovskite materials with defined properties.

Perovskite $\mathrm{CH}_{3} \mathrm{NH}_{3} \mathrm{PbI}_{3}$ was synthesized and characterized

${ }^{a}$ Centre for Clean Environment and Energy, and Griffith School of Environment, Griffith University, Gold Coast, QLD 4222, Australia; Email: h.zhao@griffith.edu.au

${ }^{b}$ Queensland Micro- and Nanotechnology Centre, Nathan Campus, Griffith University, QLD 4111, Australia

${ }^{c}$ Key Laboratory for Ultrafine Materials of Ministry of Education, School of Materials Science and Engineering, East China University of Science and technology, 130 Meilong Road, Shanghai 200237, China

${ }^{d}$ Key Laboratory of Materials Physics, Hefei Key Laboratory of Nanomaterials and Nanotechnology, Institutes of Solid State Physics, Chinese Academy of Sciences, Hefei 230031, China
35 by Weber at $1978 .{ }^{13} \mathrm{He}$ found that each $\mathrm{Pb}^{2+}$ cation is coordinated to six $\mathrm{I}^{-}$anions to form $\left[\mathrm{PbI}_{6}\right]$ octahedra, which are corner-connected to each other form a three-dimensional $\mathrm{Pb}-\mathrm{I}$ framework. Each $\mathrm{CH}_{3} \mathrm{NH}_{3}{ }^{+}$cation locates at the centre of four $\left[\mathrm{PbI}_{6}\right]$ octahedra. Thus, each cation interacts with $12 \mathrm{I}^{-}$anions. ${ }^{14-15}$ ${ }_{40}$ Previous experiments demonstrate that the symmetry and structure of $\mathrm{CH}_{3} \mathrm{NH}_{3} \mathrm{PbI}_{3}$ crystals are highly dependent on the temperature. ${ }^{15}$ At low temperature, an orthorhombic phase (space group: Pnma) is found. The orthorhombic phase transforms into a tetragonal structure (space group: $I 4 / \mathrm{m}$ ) above $161.4 \mathrm{~K}$. The cubic 45 phase (space group: $P m-3 m$ ) is observed when the temperature is higher than $330.4 \mathrm{~K}$. The improved symmetry at the higher temperature is experimentally proposed to be related to the fast dynamic movement of $\mathrm{CH}_{3} \mathrm{NH}_{3}^{+}$cations within the $\mathrm{Pb}-\mathrm{I}$ framework. As a result, the location of $\mathrm{CH}_{3} \mathrm{NH}_{3}{ }^{+}$cations can only 50 be determined experimentally in the orthorhombic phase at low temperature. ${ }^{15}$ At the same time, the lattice constants of the orthorhombic crystal vary little at the low temperature. ${ }^{15}$ Therefore, this investigation is focused on the orthorhombic perovskite $\mathrm{CH}_{3} \mathrm{NH}_{3} \mathrm{PbI}_{3}$.

55 Both structural and electronic properties of organic/inorganic hybrid perovskite $\mathrm{CH}_{3} \mathrm{NH}_{3} \mathrm{PbI}_{3}$ crystals are investigated in the present $a b$ initio calculations. Density functional theory (DFT) is employed as it offers an efficient, yet accurate quantum mechanical method for theorists to optimize structures, determine 60 energies of reactants and products, examine the nature of intermediates, and predict the reaction energies for elementary steps. ${ }^{16-19}$ However, there are still areas where traditional DFT 
with local density (LDA) or generalized gradient (GGA) approximations has its difficulties, such as the depiction of the van der Waals (vdW) interactions. ${ }^{20}$ This is because the local (LDA) or semi-local (GGA) exchange-correlation (XC) 5 functional approximations neglect the necessary ingredients to describe non-local vdW interactions. The vdW force plays an important role in a range of systems with weak interactions, such as organic/inorganic hybrid materials, particularly in their geometries. $^{21}$ Recently, GGA/DFT calculations have been 10 performed to analyse the electronic properties of cubic, tetrahedral and orthorhombic $\mathrm{CH}_{3} \mathrm{NH}_{3} \mathrm{PbI}_{3}$. In their study, the experimental structures were employed without consideration of vdW interactions. ${ }^{15}$ Thus, to get a precise theoretical understanding of organic/inorganic hybrids, methods beyond 15 traditional DFT are required.

In the last decade, empirical and less empirical approaches have been developed for prediction of $\mathrm{vdW}$ interactions in the DFT context. ${ }^{22-23}$ The vdW-DF class of dispersion energy functionals is generally considered to be a good choice for large 20 systems, except possibly for weakly bound layers, ${ }^{23-24}$ or where strongly non-additive effects are important. ${ }^{25-27}$ For the present system, a remaining issue regarding vdW-DF comes from the choice of associated semi-local exchange functionals. Here we will use a recently-proposed semi-local functional optB86b that 25 has been shown to work well in conjunction with vdW-DF for the cohesive properties of conventional solids. ${ }^{28}$ To confirm the importance of vdW effects for the properties of $\mathrm{CH}_{3} \mathrm{NH}_{3} \mathrm{PbI}_{3}$, a systematic study using local, semi-local, and non-local $\mathrm{XC}$ functionals is performed herein. We present results for fully 30 optimized structures, density of states, band structures, and charge distributions, which are compared with the available experimental data.

\section{Computation Details}

All the theoretical computations are performed by using the ${ }_{35}$ Vienna ab initio simulation package (VASP) based on DFT with the all-electron projected augmented wave (PAW) method in this study. ${ }^{29-30}$ Electron-ion interactions are described using ultrasoft pseudopotentials with a kinetic energy cut-off of $520.0 \mathrm{eV}^{31}$ Valence states included the $\mathrm{Pb} 6 \mathrm{~s}, 6 \mathrm{p}$, and $5 \mathrm{~d}$ states, I $5 \mathrm{~s}$ and $5 \mathrm{p}$
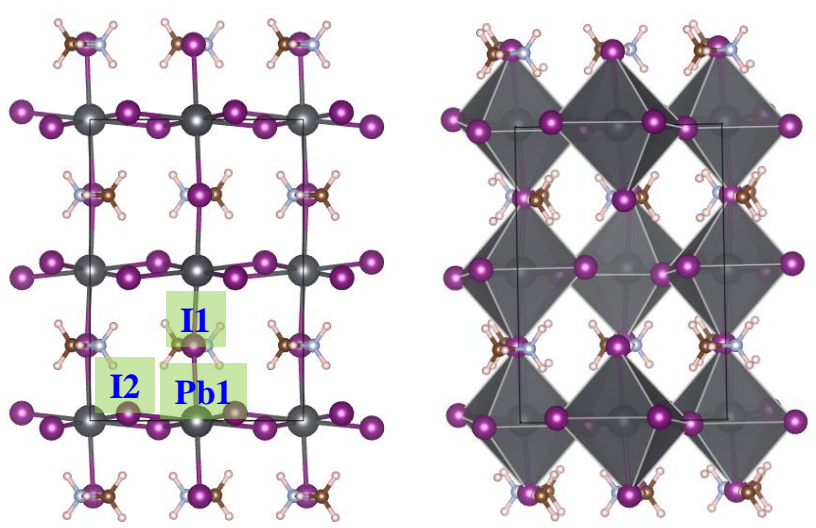

Fig. 1 Atomic structures of orthorhombic perovskite $\mathrm{CH}_{3} \mathrm{NH}_{3} \mathrm{PbI}_{3}$ crystals without (left) or with (right) showing the $\left[\mathrm{PbI}_{6}\right]$ octahedra. Key: grey- $\mathrm{Pb}$, purple- I, light blue- N, brown- $\mathrm{C}$, and pink- $\mathrm{H}$.
Table 1 The theoretical lattice constants and $\mathrm{Pb}-\mathrm{I}$ bond lengths of orthorhombic $\mathrm{CH}_{3} \mathrm{NH}_{3} \mathrm{PbI}_{3}$ and $\mathrm{CsPbI}_{3}$ in comparison with the experimental data.

\begin{tabular}{ccccc}
\hline & $a(\AA)$ & $b(\AA)$ & $c(\AA)$ & $\mathrm{V}\left(\AA^{3}\right)$ \\
\hline PZ81/LDA & 8.678 & 12.387 & 8.318 & 894.05 \\
PBE/GGA & 9.226 & 12.876 & 8.619 & 1023.88 \\
optB86b+vdWDF & 8.831 & 12.648 & 8.570 & 957.18 \\
Exp ${ }^{15}$ & 8.836 & 12.580 & 8.555 & 951.01 \\
Exp ${ }^{14}$ & 8.861 & 12.659 & 8.581 & 962.54 \\
\hline & & $\mathrm{CsPbI}_{3}$ & & 820.92 \\
PZ81/LDA & 4.674 & 10.17 & 17.27 & 868.09 \\
PBE/GGA & 4.909 & 10.80 & 18.26 & 888.97 \\
optB86b+vdWDF & 4.793 & 10.45 & 17.74 & 17.76 \\
Exp ${ }^{37}$ & 4.795 & 10.45 & & 889.91 \\
\hline
\end{tabular}

40 states, C $2 \mathrm{~s}$ and $2 \mathrm{p}$ states, $\mathrm{N} 2 \mathrm{~s}$ and $2 \mathrm{p}$ states, and the $\mathrm{H} 1 \mathrm{~s}$ states. When the geometry is optimized, all atoms are allowed to relax. The cell optimization technique is employed to optimize the lattice constants. We perform Brillouin-zone integrations using Monkhorst-Pack grids of special points with $(4 \times 4 \times 4)$ and $45(8 \times 8 \times 8)$ meshes for the calculations of structural and electronic properties, respectively. The supercell of orthorhombic $\mathrm{CH}_{3} \mathrm{NH}_{3} \mathrm{PbI}_{3}$ includes 48 atoms.

A range of specific functionals have been developed for local LDA, semi-local GGA, and non-local vdW-DF approaches 50 previously. In this study, typical functionals for each approach have been chosen to represent their respective class. The functionals parameterized by $(\mathrm{PZ} 81)^{32}$ and Perdew-BurkeErnzerhhof $(\mathrm{PBE})^{33}$ are employed for local LDA and semi-local GGA methods, respectively. Here, we use the non-local vdW-DF 55 method proposed by Dion et al. to describe the vdW interaction as modified by Klimes et al.. ${ }^{23,28}$ In this approach, the XC energy $E_{X C}$ takes the form:

$$
E_{X C}=E_{X}^{G G A}+E_{C}^{L D A}+E_{C}^{n l}
$$

Here the exchange energy $E_{X}^{G G A}$ uses the optimized optB86b GGA ${ }_{60}$ functional, ${ }^{28}$ and $E_{C}^{L D A}$ is the LDA functional for correlation energy. $E_{C}^{n l}$ is obtained using a relatively simple double space integration.

\section{Results and discussion}

${ }_{65}$ Determining correct structural properties of materials is essential for the analysis of their other properties, such as the bandgap of semiconductors. ${ }^{34}$ To compare the performance of various functionals, the structures of organic/inorganic hybrid perovskite $\mathrm{CH}_{3} \mathrm{NH}_{3} \mathrm{PbI}_{3}$ crystals are firstly optimized. The initial 70 lattice constants and atomic coordinates of orthorhombic $\mathrm{CH}_{3} \mathrm{NH}_{3} \mathrm{PbI}_{3}$ crystals for theoretical optimizations are from the $\mathrm{X}$-ray diffraction (XRD) experiments at $100 \mathrm{~K}^{15}$ The optimized lattice constants and volumes using the various functionals are listed in Table 1 with the available experimental values. ${ }^{14-15}$ In 75 general, the PZ81/LDA functional underestimates all the lattice constants, while PBE/GGA functional overestimates them. ${ }^{35}$ The 
Table 2 The displacements parameters of $\mathrm{Pb} 1, \mathrm{I} 1$ and $\mathrm{I} 2$ atoms for orthorhombic $\mathrm{CH}_{3} \mathrm{NH}_{3} \mathrm{PbI}_{3}$.

\begin{tabular}{|c|c|c|c|c|}
\hline & & $U_{x}$ & $U_{y}$ & $U_{z}$ \\
\hline \multirow[t]{4}{*}{$\mathrm{Pb} 1$} & PZ81 & 0.000 & 0.000 & 0.000 \\
\hline & PBE & 0.000 & 0.002 & 0.002 \\
\hline & optB86b+vdWDF & 0.001 & 0.000 & 0.000 \\
\hline & $\operatorname{Exp}^{15}$ & 0.007 & 0.003 & 0.009 \\
\hline \multirow[t]{4}{*}{ I1 } & PZ81 & 0.020 & 0.000 & 0.060 \\
\hline & PBE & 0.014 & 0.002 & 0.064 \\
\hline & optB86b+vdWDF & 0.023 & 0.000 & 0.065 \\
\hline & $\operatorname{Exp}^{15}$ & 0.019 & 0.002 & 0.018 \\
\hline \multirow[t]{4}{*}{$\mathrm{I} 2$} & PZ81 & 0.013 & 0.016 & 0.007 \\
\hline & PBE & 0.015 & 0.024 & 0.013 \\
\hline & optB86b+vdWDF & 0.015 & 0.017 & 0.010 \\
\hline & $\operatorname{Exp}^{15}$ & 0.016 & 0.021 & 0.014 \\
\hline
\end{tabular}

deviations of volumes of PZ81 and PBE are larger than $6.0 \%$. The variations of the theoretical lattice constants using PZ81 and PBE are close to those of other solids using similar functionals. ${ }^{36}$ In our calculation, the best structural descriptions are obtained 5 with the non-local optB86b+vdWDF functional. The deviation with respect to the experimental data is less than $1.0 \%$. To detect the origin of the improvement, we also calculate the structural properties of orthorhombic $\mathrm{CsPbI}_{3}$ crystals, as listed in Table 1. It can be found that properties determined using PBE/GGA and 10 optB86b + vdWDF methods are similar as that of orthorhombic $\mathrm{CH} 3 \mathrm{NH} 3 \mathrm{PbI} 3{ }^{37}$ Considering that the main different between $\mathrm{CsPbI}_{3}$ and $\mathrm{CH}_{3} \mathrm{NH}_{3} \mathrm{PbI}_{3}$ crystals are the cations. Thus, the significant improvement on the calculation accuracy of the orthorhombic $\mathrm{CH}_{3} \mathrm{NH}_{3} \mathrm{PbI}_{3}$ crystals demonstrates that the weak

$15 \mathrm{vdW}$ binding between the organic components across the inorganic framework is required to depict the structural properties for this type of organic/inorganic hybrid materials. This conclusion is different from the assumption used in the recent theoretical studies on organic/inorganic hybrid tetragonal 20 perovskite materials. ${ }^{38}$ The optimized atomic structure of the orthorhombic $\mathrm{CH}_{3} \mathrm{NH}_{3} \mathrm{PbI}_{3}$ crystals using the optB86b+vdWDF functional is shown in Fig.1.

The structures from the XRD refinement experiments show angular distortions of the $\left[\mathrm{PbI}_{6}\right]$ octahedra, ${ }^{15}$ such as $\mathrm{I}^{-}$anions 25 have a significant transverse displacement from the mid-point of the $\mathrm{Pb}-\mathrm{Pb}$ distance to which they are constrained in the ideal crystallographic description (see Fig. 1). The displacements of atoms cause a reduction in symmetry of the $\mathrm{CH}_{3} \mathrm{NH}_{3} \mathrm{PbI}_{3}$ crystal from the cubic phase to the orthorhombic phase. Table 2 lists the 30 theoretical displacement parameters of $\mathrm{Pb} 1, \mathrm{I} 1$ and $\mathrm{I} 2$ atoms, marked in Fig. 1, using various functionals compared with the experimental data. The displacement parameters are the differences of the fractional coordinates of atoms between optimized structures and ideal crystallographic structures. It can

35 be seen that most of the theoretical displacement parameters are in very good agreement with the experimental values, except the displacement of I1 atoms along the $c$ direction. ${ }^{15}$ Also, the
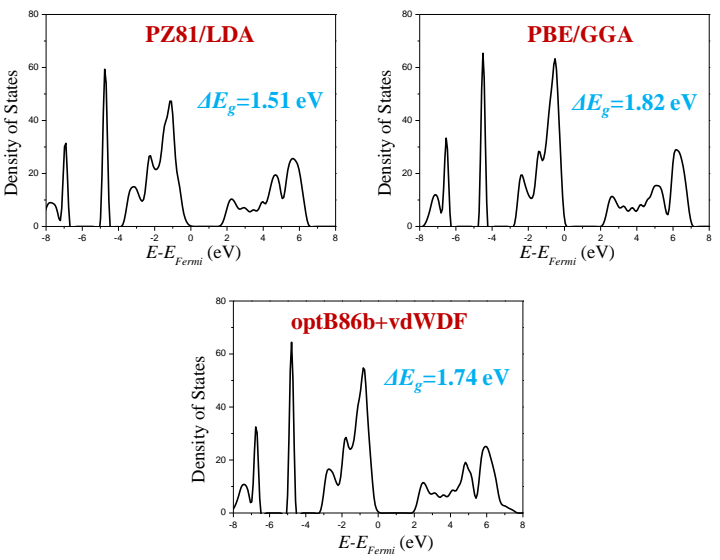

Fig. 2 Total density of states (TDOS) of orthorhombic $\mathrm{CH}_{3} \mathrm{NH}_{3} \mathrm{PbI}_{3}$ crystals and their bandgap energies using different functionals.

displacements of the I1 atoms in $a$ and $c$ directions are larger than those of the I2 atoms. The I2 atoms shift more along the $b$ 40 direction. The $\mathrm{Pb}-\mathrm{I} 1-\mathrm{Pb}$ angle is about $159^{\circ}$ in optB86b+vdWDF results, which is $11^{\circ}$ larger than the $\mathrm{Pb}-\mathrm{I} 2-\mathrm{Pb}$ angle. These structural differences indicate that I1 and I2 atoms are inequivalent atoms.

Since the organic/inorganic hybrid perovskite $\mathrm{CH}_{3} \mathrm{NH}_{3} \mathrm{PbI}_{3}$ 45 materials are used as light harvesters in DSCs, ${ }^{4-6,} 10$ their electronic structures are crucial factors for sunlight absorption. In this regard, the total density of states (TDOS) is calculated using the various functionals (see Fig. 2). In our calculations, the bandgap energy with the PZ81/LDA functional is $1.51 \mathrm{eV}$, which 50 is $17.9 \%$ less than that with the PBE/GGA functional. Our bandgap energy of the PBE/GGA functional is ca. $10 \%$ larger than the previous theoretical value. ${ }^{15}$ This is because the lattice constants used in our study are $8.0 \%$ larger than the previous one, in which the experimental lattice constants were used. Our results 55 support the strong relationship between structural properties and bandgap energies, and highlight the importance of using approximations which accurately reproduce the correct geometry. ${ }^{34}$ When the optB86b+vdWDF functional is employed, the theoretical bandgap energy is $1.74 \mathrm{eV}$, which is ca. $0.1 \mathrm{eV}$ 60 smaller than that with the PBE functional. This difference can also be ascribed to the different theoretical lattice constants given by various functionals. According to the optical absorption experiments, the electronic bandgap of orthorhombic $\mathrm{CH}_{3} \mathrm{NH}_{3} \mathrm{PbI}_{3}$ crystals is $1.68-1.72 \mathrm{eV}$ at low temperature. ${ }^{39-40}$ ${ }_{65}$ Consequently, the experimental bandgap energies of hybrid $\mathrm{CH}_{3} \mathrm{NH}_{3} \mathrm{PbI}_{3}$ are surprisingly close to the theoretical predictions at the semi-local and non-local level. Previous DFT studies demonstrate that the bandgap energies of solid-state semiconductors are seriously underestimated by using pure DFT 70 functionals. In some cases, the deviation is about $30 \% .^{41-42}$ This is because good band gaps are not normally to be expected from functionals of the types tested here, which do not have hybrid characters. $^{42-44}$ This successful match is likely to be fortuitous, which is also observed in other Pb-based materials. ${ }^{15,38,45}$ Fig. 2 75 also shows that that the characteristics of peaks using various functionals in TDOS images are broadly similar, except for the locations of the peaks. 

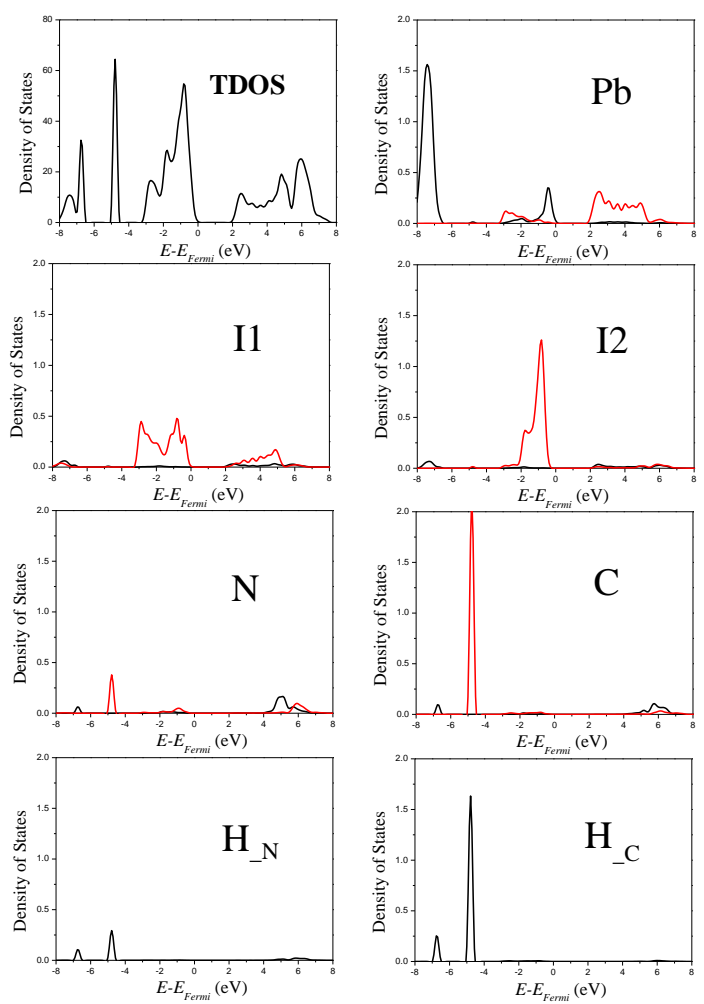

Fig. 3 TDOS and Partial density of states (PDOS) of atoms in orthorhombic $\mathrm{CH}_{3} \mathrm{NH}_{3} \mathrm{PbI}_{3}$ crystals using the optB86b+vdWDF functional. The black and red lines are for $\mathrm{s}$ and $\mathrm{p}$ states, respectively.

To understand the bonding mechanisms between the atoms, the analysis of partial density of states (PDOS) has been performed. The calculations of TDOS reveal that the functionals have small effects on the main characteristics of peaks. Therefore, 5 only the analysis of PDOS using the optB86b+vdWDF functional is shown in Fig. 3. The PDOS images of I1 and I2 supports the argument that these two kinds of I atoms are chemically inequivalent. The PDOS peaks of I2 atoms are closer to the Fermi energy and sharper than those of I1 atoms in the top valence 10 bands. The stronger hybridization of the orbitals can shift the location of valence bands to the lower energy area with wider peaks. ${ }^{46}$ Thus, the different PDOS images of I1 and I2 atoms indicate a weaker bonding between $\mathrm{Pb}$ and $\mathrm{I} 2$ atoms. From Fig. 3, organic $\mathrm{CH}_{3} \mathrm{NH}_{3}{ }^{+}$cations have little contribution to the top 15 valence and bottom conduction bands around the Fermi energy level $\left(-4.0 \mathrm{eV}<E-E_{F e r m i}<4.0 \mathrm{eV}\right)$. The main contribution to the top valence band is from the I $5 \mathrm{p}$ states with an overlapping of $\mathrm{Pb}$ $6 \mathrm{~s}$ states. In the bottom conduction bands, the main components are $\mathrm{Pb} 6 \mathrm{p}$ states. The PDOS images suggest that I $5 \mathrm{p}$ electrons, 20 especially the $\mathrm{I} 25 \mathrm{p}$ electrons, can be photo-excited to $\mathrm{Pb} 6 \mathrm{p}$ empty states. Consequently, I atoms change into photohole sites and $\mathrm{Pb}$ atoms hold the photoelectrons after the photo-excitation. From Fig. 3, we also find that the highest $\mathrm{C}$ and $\mathrm{N} 2 \mathrm{p}$ bands and $\mathrm{H} 1 \mathrm{~s}$ bands locate at -6.8 and $-4.8 \mathrm{eV}$, which has little overlap 25 with the $\mathrm{Pb}$ and I orbitals. Thus, there is no covalent interaction between the organic cations and the inorganic $\mathrm{Pb}-\mathrm{I}$ framework.

The band structures of orthorhombic $\mathrm{CH}_{3} \mathrm{NH}_{3} \mathrm{PbI}_{3}$ using

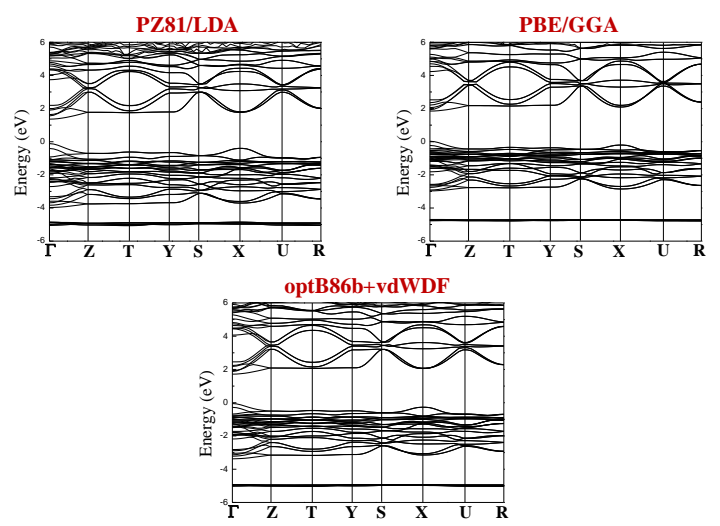

Fig. 4 Calculated band structure of orthorhombic $\mathrm{CH}_{3} \mathrm{NH}_{3} \mathrm{PbI}_{3}$ crystals along the high-symmetry lines in the first Brillouin zone using PZ81/LDA, PBE/GGA, and optB86b+vdWDF functionals, respectively.

different functionals are shown in Fig. 4. Our results agree with previous studies in showing that the orthorhombic $\mathrm{CH}_{3} \mathrm{NH}_{3} \mathrm{PbI}_{3}$ is 30 a direct-bandgap crystal with the minimum band gap at the $\Gamma$ symmetry point. ${ }^{15}$ The minimum indirect bandgap is from the $\Gamma$ point to the $\mathrm{X}$ point. For all band structures, several sets of bands can be found. They correspond to $\mathrm{C} 2 \mathrm{p}$ and $\mathrm{H}_{-\mathrm{C}}$ (the $\mathrm{H}$ atoms bonded to $\mathrm{C}$ atoms) $1 \mathrm{~s}$ states around $-5 \mathrm{eV}$, mainly I $5 \mathrm{p}$ states 35 below the Fermi level, and $\mathrm{Pb} 6 \mathrm{p}$ states above the Fermi level. Both the top valence band and the bottom conduction band are broad, indicating these states are non-localized. This explains why the excitons can be transported long distance in this material. ${ }^{11-12}$ Moreover, the fluctuations of the states in the

40 bottom conduction band are stronger, indicating a faster transport of photoelectrons that matches the experimental observations. ${ }^{12}$ On the contrary, the band for organic fragments (the band around $-5 \mathrm{eV}$ ) is flat and narrow, suggesting that the electrons around them are localized. It should also be noted that the band structure 45 of the orthorhombic $\mathrm{CH}_{3} \mathrm{NH}_{3} \mathrm{PbI}_{3}$ crystal is quite different from that of cubic phase due to the lower symmetry and bigger primitive cell. The main functional dependent properties are the locations of the bands, which confirm the conclusion from the analysis of DOS images.

50 The Bader charges of $\mathrm{Pb}, \mathrm{I} 1, \mathrm{I} 2, \mathrm{~N}$ and $\mathrm{C}$ atoms in the

Table 3. Badar atomic charges of $\mathrm{CH}_{3} \mathrm{NH}_{3} \mathrm{PbI}_{3}$ using various functionals

\begin{tabular}{ccccc}
\hline & LDA & PBE & optB86b+vdWDF & $\begin{array}{c}\text { optB86b+vdWDF } \\
(\mathrm{AE})\end{array}$ \\
\hline $\mathrm{Pb}$ & +0.85 & +0.95 & +0.92 & +0.86 \\
$\mathrm{I} 1$ & -0.52 & -0.57 & -0.55 & -0.55 \\
$\mathrm{I} 2$ & -0.50 & -0.55 & -0.54 & -0.54 \\
$\mathrm{~N}$ & -3.01 & -2.96 & -2.95 & -1.33 \\
$\mathrm{C}$ & +0.35 & +0.53 & +0.48 & +0.44 \\
$\mathrm{PbI}_{3}$ & -0.67 & -0.72 & -0.70 & -0.77 \\
$\mathrm{CH}_{3}$ & +0.67 & +0.67 & +0.64 & +0.68 \\
$\mathrm{NH}_{3}$ & -0.01 & +0.04 & +0.05 & +0.02 \\
\hline
\end{tabular}


orthorhombic perovskite $\mathrm{CH}_{3} \mathrm{NH}_{3} \mathrm{PbI}_{3}$ crystals based on the pseudo valence density using different functionals are listed Table 3. In general, the charge analysis provides a picture of ionic interactions between inorganic $\mathrm{Pb}$-I framework and organic ${ }_{5} \mathrm{CH}_{3} \mathrm{NH}_{3}{ }^{+}$cations since the average charge per $\mathrm{PbI}_{3}$ and $\mathrm{CH}_{3} \mathrm{NH}_{3}$ unit is ca. -0.7 e and +0.7 e. On the contrary, charges of $\mathrm{Pb}$ and $\mathrm{I}$ atoms correspond to significant deviations from purely ionic interactions (i.e., $\mathrm{Pb}^{2+}$ and $\mathrm{I}^{-}$), which suggests a combined covalent and ionic bonding mechanism between $\mathrm{Pb}$ and I atoms.

10 The $\mathrm{Pb}-\mathrm{I}$ covalent bonding characteristics can also be supported by the hybridization of the $\mathrm{Pb} 6 \mathrm{~s}, 6 \mathrm{p}$ states with the I $5 \mathrm{p}$ states at the top valence bands shown in Fig. 3. Within $\mathrm{CH}_{3} \mathrm{NH}_{3}{ }^{+}$cations, the $\mathrm{NH}_{3}$ group is almost charge neutral; and most of positive charge is the contribution from the $\mathrm{CH}_{3}$ group. This charge 15 distribution matches the chemical instinct because $\mathrm{NH}_{3}$ is a charge neutral molecule and $\mathrm{CH}_{3}$ is a positively charge cation in the gas phase. The charge distribution shown in Table 3 indicates that there is a slightly stronger ionic interaction between $\mathrm{CH}_{3}$ group and I1 atoms. From the PDOS analysis, there is a stronger ${ }_{20} \mathrm{~Pb}-\mathrm{I} 1$ covalent bonding. Thus, I1 atoms have overall stronger interactions with their adjacent atoms, which explains why I1 and I 2 atoms are chemically inequivalent, as observed in Fig. 3. In the tetragonal and cubic phase, the symmetries of $\mathrm{Pb}$-I frameworks are improved due to the thermal movement of organic cations. ${ }^{15}$

25 In those cases, the ineqivalence of I atoms may be eliminated. A study on the thermal effect on the phase change and properties of $\mathrm{CH}_{3} \mathrm{NH}_{3} \mathrm{PbI}_{3}$ materials is currently under way. Since the charge analysis using the all-electron (AE) reconstructed valence density could give more accurate results, ${ }^{47}$ the calculations based on the $30 \mathrm{AE}$ data with optB86B+vdWDF are also performed, as listed in Table 3. It can be seen that conclusions drawn from both sets of data are almost identical. The main difference is the charge of $\mathrm{N}$ atoms and the $\mathrm{H}$ atoms bonded with $\mathrm{N}$ atoms.

\section{4. Conclusions}

In summary, first principle DFT calculations have been performed to study structural and electronic properties of orthorhombic perovskite $\mathrm{CH}_{3} \mathrm{NH}_{3} \mathrm{PbI}_{3}$ materials. Our results demonstrate that the employment of optB86b+vdWDF XC 40 functional improves the accuracy of the calculated structural properties significantly compared with the non-vdW functionals employed here. Thus, consideration of the vdW interaction is important for theoretical studies of this organic/inorganic hybrid material. The main interaction between the organic groups and 45 inorganic framework is through the ionic binding between $\mathrm{CH}_{3}{ }^{+}$ cations and $\mathrm{I}^{-}$anions. The different interaction strengths between I atoms with their adjacent atoms cause the formation of two kinds of I atoms in the $\mathrm{Pb}$-I framework. Analysis of the electronic properties supports the conclusion that orthorhombic ${ }_{50} \mathrm{CH}_{3} \mathrm{NH}_{3} \mathrm{PbI}_{3}$ is a direct-bandgap crystal with the minimum band gap at the $\Gamma$ symmetry point. During light harvesting, I $5 p$ electrons can be photo-excited to $\mathrm{Pb} 6 \mathrm{p}$ empty states. Our results, therefore, pave a way for the further theoretical studies on this type of organic/inorganic hybrid perovskite materials for 55 sensitized solar cells.

\section{Acknowledgements}

We thank the Australian Research Council for funding. This research was undertaken on the National Computational ${ }_{60}$ Infrastructure (NCI) in Canberra, Australia, which is supported by the Australian Commonwealth Government.

\section{Notes and references}

1. M. Gratzel, Nature, 2001, 414, 338-344.

2. B. Oregan and M. Gratzel, Nature, 1991, 353, 737-740.

65 3. S. F. Zhang, X. D. Yang, Y. H. Numata and L. Y. Han, Energy Environ. Sci., 2013, 6, 1443-1464.

4. J. Burschka, N. Pellet, S. J. Moon, R. Humphry-Baker, P. Gao, M. K. Nazeeruddin and M. Gratzel, Nature, 2013, 499, 316-320.

5. J. Bisquert, J. Phys. Chem. Lett., 2013, 4, 2597-2598.

706 . H. S. Kim, C. R. Lee, J. H. Im, K. B. Lee, T. Moehl, A. Marchioro, S. J. Moon, R. Humphry-Baker, J. H. Yum, J. E. Moser, M. Gratzel and N. G. Park, Sci Rep-Uk, 2012, 2, 591.

7. M. M. Lee, J. Teuscher, T. Miyasaka, T. N. Murakami and H. J. Snaith, Science, 2012, 338, 643-647.

75 8. N. G. Park, J Phys Chem Lett, 2013, 4, 2423-2429.

9. D. Q. Bi, L. Yang, G. Boschloo, A. Hagfeldt and E. M. J. Johansson, J Phys Chem Lett, 2013, 4, 1532-1536.

10. M. Liu, M. B. Johnston and H. J. Snaith, Nature, 2013, 501, 395-398.

11. S. D. Stranks, G. E. Eperon, G. Grancini, C. Menelaou, M. J.

80 Alcocer, T. Leijtens, L. M. Herz, A. Petrozza and H. J. Snaith, Science, 2013, 342, 341-344.

12. G. Xing, N. Mathews, S. Sun, S. S. Lim, Y. M. Lam, M. Gratzel, S. Mhaisalkar and T. C. Sum, Science, 2013, 342, 344-347.

13. D. Weber, Z Naturforsch B, 1978, 33, 1443-1445.

85 14. A. Poglitsch and D. Weber, J Chem Phys, 1987, 87, 6373-6378.

15. T. Baikie, Y. N. Fang, J. M. Kadro, M. Schreyer, F. X. Wei, S. G. Mhaisalkar, M. Graetzel and T. J. White, J Mater Chem A, 2013, 1, 5628-5641.

16. W. Kohn and L. J. Sham, Phys. Rev. A, 1965, 140, 1133.

90 17. Y. Zhao, N. E. Schultz and D. G. Truhlar, J. Chem. Theory Comput., 2006, 2, 364-382

18. A. Vittadini, M. Casarin and A. Selloni, Theor. Chem. Acc., 2007, 117, 663-671.

19. E. A. Carter, Science, 2008, 321, 800-803.

95 20. Y. Andersson, D. C. Langreth and B. I. Lundqvist, Phys Rev Lett, 1996, 76, 102-105.

21. J. P. P. Ramalho, J. R. B. Gomes and F. Illas, Rsc Adv, 2013, 3, 13085-13100.

22. S. Grimme, J. Antony, S. Ehrlich and H. Krieg, J Chem Phys, 2010, $100 \quad 132$

23. M. Dion, H. Rydberg, E. Schroder, D. C. Langreth and B. I. Lundqvist, Phys Rev Lett, 2004, 92, 246401.

24. T. Bjorkman, A. Gulans, A. V. Krasheninnikov and R. M. Nieminen, Phys Rev Lett, 2012, 108, 235502.

105 25. J. F. Dobson, A. White and A. Rubio, Phys Rev Lett, 2006, 96, 073201.

26. J. F. Dobson and T. Gould, J Phys-Condens Mat, 2012, 24.

27. V. V. Gobre and A. Tkatchenko, Nat Commun, 2013, 4, 2341.

28. J. Klimes, D. R. Bowler and A. Michaelides, Phys Rev B, 2011, 83, $110 \quad 195131$

29. G. Kresse and J. Furthmuller, Comput. Mater. Sci., 1996, 6, 15-50.

30. G. Kresse and D. Joubert, Phys. Rev. B, 1999, 59, 1758-1775.

31. D. Vanderbilt, Phys. Rev. B, 1990, 41, 7892. 
32. J. P. Perdew and A. Zunger, Phys. Rev. B, 1981, 23, 5048.

33. J. P. Perdew, K. Burke and M. Ernzerhof, Phys Rev Lett, 1996, 77, 3865-3868.

34. I. Borriello, G. Cantele and D. Ninno, Phys Rev B, 2008, 77, 235214.

5 35. A. E. Mattsson, R. Armiento, J. Paier, G. Kresse, J. M. Wills and T. R. Mattsson, J Chem Phys, 2008, 128, 084714.

36. G. I. Csonka, J. P. Perdew, A. Ruzsinszky, P. H. T. Philipsen, S. Lebegue, J. Paier, O. A. Vydrov and J. G. Angyan, Phys Rev $B, 2009,79,155107$.

10 37. C. K. Moller, Nature, 1958, 182, 1436-1436.

38. E. Mosconi, A. Amat, M. K. Nazeeruddin, M. Gratzel and F. De Angelis, J Phys Chem C, 2013, 117, 13902-13913.

39. T. Ishihara, J Lumin, 1994, 60-1, 269-274.

40. G. C. Papavassiliou and I. B. Koutselas, Synth. Met., 1995, 71, 1713-

15 1714.

41. Y. Wang, T. Sun, D. J. Yang, H. W. Liu, H. M. Zhang, X. D. Yao and H. J. Zhao, Phys. Chem. Chem. Phys., 2012, 14, 23332338.

42. Y. Wang, H. M. Zhang, P. R. Liu, X. D. Yao and H. J. Zhao, RSC

20 Adv., 2013, 3, 8777-8782.

43. J. Heyd, G. E. Scuseria and M. Ernzerhof, J. Chem. Phys., 2006, 124, 2204597.

44. Y. Wang, S. de Gironcoli, N. S. Hush and J. R. Reimers, J. Am. Chem. Soc., 2007, 129, 10402-10407.

25 45. H. Z. Lv, H. W. Gao, Y. Yang and L. K. Liu, Appl Catal a-Gen, 2011, 404, 54-58.

46. R. Hofmann, Solids and surfaces: A chemist's view of bonding in extended structures, VCH publishers, Inc., New York, 1988.

47. E. Aubert, S. Lebegue, M. Marsman, T. T. B. Thai, C. Jelsch, S. Dahaoui, E. Espinosa and J. G. Angyan, J Phys Chem A, 2011, 115, 14484-14494. 